\title{
O PERFIL DOS EGRESSOS DO CURSO DE ENGENHARIA CIVIL DO CCT/UDESC: A IMPORTÂNCIA NA REFORMULAÇÃO DO PPC
}

DOI: 10.37702/2175-957X.COBENGE.2021.3486

Jennifer Perreira dos Santos - sjenniferp@gmail.com

Universidade do Estado de Santa Catarina

Rua Ibrahim Manoel Matos 90

89230-550 - Joinville - SC

SANDRA DENISE KRUGER ALVES - sandra.kruger@udesc.br

Universidade do Estado de Santa Catarina

RUA JOAO PESSOA 984

89218-280 - JOINVILLE - SC

Leonardo Romero Monteiro - leonardo.monteiro@udesc.br

Universidade do Estado de Santa Catarina

Rua Dom Bosco 155

89222-540 - Joinville - SC

Resumo: Para que um curso de graduação consiga atingir os seus objetivos de desenvolvimento de competências, um currículo integrado deve ser concebido através da coletividade. Dessa forma, é imprescindível que se levantem os dados do curso no intuito de obter demandas de formação técnica e pedagógica para dar suporte ao novo currículo a ser implementado. O presente trabalho buscou investigar, sob a perspectiva do egresso, as potencialidades e fragilidades do curso de Engenharia Civil do CCT/UDESC. Para isso, o Núcleo Docente Estruturante (NDE) do curso elaborou um formulário que foi aplicado aos alunos formados entre 2016/1 e 2020/1. Este formulário obteve respostas de 44\% dos egressos contatados. A partir das respostas registradas foi desenvolvido um banco de dados dos egressos do curso e elaborado um painel das respostas com a finalidade de auxiliar os membros do NDE na concepção do novo PPC. Ao longo das atividades descritas nesse trabalho percebeu-se carência de informações a respeito da análise de egressos de cursos de engenharia e, portanto, conferiu relevância a pesquisa realizada.

Palavras-chave: Ensino em Engenharia. Diretrizes Curriculares Nacionais. Projeto Pedagógico de Curso. Perfil do egresso. 


\section{O PERFIL DOS EGRESSOS DO CURSO DE ENGENHARIA CIVIL DO CCT/UDESC: A IMPORTÂNCIA NA REFORMULAÇÃO DO PPC}

\section{INTRODUÇÃO}

A partir da última década, transformações pedagógicas nas estruturas curriculares da educação superior passaram a indicar a necessidade de novas práticas de ensino voltadas à construção dos saberes fundamentais, competências e habilidades (MARINHOARAUJO; RABELO, 2015). Nesse contexto, as novas Diretrizes Curriculares Nacionais DCNs (CNE/CES, 2019) são baseadas nas necessidades de formação profissional da sociedade e por isso fomentam que os egressos dos cursos de engenharia devem estar aptos a criar, projetar e analisar produtos, processos, além de serem capazes de implantar, supervisionar e controlar as soluções propostas respeitando a normatização pertinente e a ética do exercício profissional (PRADO; SANTOS, 2019).

Para que um currículo integrado seja concebido é preciso que exista coletividade durante a sua construção, caso contrário muito dificilmente as competências necessárias para o perfil do egresso serão desenvolvidas ao final do processo. Dessa forma, é imprescindível que se levantem dados do curso através de um processo de autoavaliação no intuito de obter demandas de formação técnica e pedagógica para dar suporte ao novo currículo a ser implementado.

\section{O CURSO DE GRADUAÇÃO EM ENGENHARIA CIVIL DO CENTRO DE CIÊNCIAS TECNOLÓGICAS DA UNIVERSIDADE DO ESTADO DE SANTA CATARINA}

O atual curso de Engenharia Civil do campus de Joinville da UDESC (Centro de Ciências Tecnológicas) foi criado em 1979 e o projeto pedagógico vigente foi publicado em 2008 (DEC UDESC/CCT, 2008), devendo ser atualizado até 2023 para respeitar as novas DCNs publicadas em 2019. Segundo a proposta do PPC de 2008 o curso possui 4968 horas-aula, que são distribuídas em 10 semestres com 18 semanas cada (DEC UDESC/CCT, 2008) englobando disciplinas obrigatórias, disciplinas optativas, Trabalho de Conclusão de Curso (TCC), Estágio Curricular Supervisionado (ECS) e atividades complementares. De acordo com este PPC, o curso de Engenharia Civil objetiva desenvolver a consciência social e política do aluno, de forma a capacitá-lo para o exercício da cidadania plena. São, também, objetivos da formação proporcionada pelo curso:

I. Proporcionar uma sólida formação em disciplinas básicas e profissionalizantes que habilitem o egresso a acompanhar o ritmo de desenvolvimento técnico-científico do setor e adaptar-se às transformações;

II. Estimular o espírito de iniciativa, inventividade, empreendedorismo e de liderança, capacitando o profissional como agente ativo das transformações sociais;

III. Estimular um bom relacionamento humano, com posturas éticas e de colaboração permanente;

IV. Desenvolver a capacidade de comunicação gráfica, oral e escrita, sabendo utilizar as ferramentas necessárias para um melhor desempenho das funções;

V. Colaborar com uma postura relacionada com o respeito ao meio ambiente, à segurança do trabalhador, e a sensibilidade relativa a aspectos sociais e a sustentabilidade. 


\section{O NÚCLEO DOCENTE ESTRUTURANTE DO CURSO}

Os membros do Núcleo Docente Estruturante (NDE) são os professores responsáveis, entre outras ações, pelas reformulações necessárias na estrutura do curso e, consequentemente, da elaboração de um novo PPC. Por esse motivo, a partir da metade do ano de 2020 os membros foram convidados a iniciarem as discussões sobre a reestruturação do PPC vigente. Nestas reuniões os membros do NDE foram introduzidos aos aspectos das novas DCNs.

Foram incentivados encontros mensais do grupo do NDE para iniciar a reformulação do PPC. Em uma primeira reunião foram apresentados estudos realizados para 0 reconhecimento do curso e compreensão da nova DCN para que, deste modo, os docentes se sensibilizassem e percebessem a situação atual do curso de graduação e quais são as tendências de mudanças no ensino superior. As reuniões seguintes deram continuidade ao que foi levantado nas reuniões anteriores, bem como inseriram à discussão novos aspectos a serem considerados no desenvolvimento do desenho do novo PPC.

Em uma destas reuniões foi levantada a ideia de formular uma autoavaliação através de uma investigação da opinião do egresso sobre o curso de Engenharia Civil do CCT/UDESC com a perspectiva de auxiliar o NDE no entendimento do potencial do curso e contribuiria na definição de uma nova estrutura curricular em consonância aos pontos estudados e levantados na DCN de 2019. Para tal fim, três membros do grupo se encarregaram de realizar o procedimento utilizando um formulário on-line, nesse caso o Microsoft Forms.

\section{FORMULÁRIO APLICADO AOS EGRESSOS}

O formulário aplicado objetivou capturar a percepção dos egressos investigando, na sua visão, as potencialidades e fragilidades do curso de Engenharia Civil do CCT/UDESC. Assim, esta pesquisa pôde elucidar, através do estudo do perfil do egresso, algumas sugestões de mudanças para a elaboração de um novo currículo.

O formulário foi encaminhado para os alunos graduados no período compreendido entre 2016/1 e 2020/1 totalizando 302 alunos dos quais 301 foram contatados através do e-mail de cadastro na universidade e/ou redes sociais. Ressalta-se que a escolha do período a partir de 2016/1 se deve ao fato de coincidir com a primeira turma de formados após a última reforma do curso, ou seja, segundo o PPC vigente. Este formulário ficou disponível para respostas entre os dias 05/12/2020 e 20/12/2020. Ao fim deste período foram extraídas 133 respostas registradas configurando uma participação de aproximadamente $44 \%$ dos egressos inicialmente contatados.

O formulário foi composto de 37 questões sendo 4 questões de identificação do egresso, 23 questões objetivas e 10 questões discursivas. As questões de identificação tinham o intuito de construir um banco de dados dos egressos e solicitavam nome, e-mail, telefone de contato, ano/semestre de ingresso e conclusão do curso. O objetivo da criação de um banco de dados dos egressos e, consequentemente, do estabelecimento de uma política de egressos foi de manter contato com os formados e aproximá-los das atividades da universidade mesmo após a conclusão do curso. Além disso, o acompanhamento dos egressos do curso permitiu revelar as áreas consolidadas do curso, bem como áreas deficitárias.

As questões objetivas, por sua vez, buscaram mensurar as respostas estabelecendo classificações para diversos aspectos do curso mediante a utilização de escalas Likert, como exemplificado pelas Figuras 1 e 2. Desse modo, os respondentes podiam classificar 
a sua experiência de acordo com as opções e assim foram abordados alguns pontos relevantes à compreensão da situação atual do curso.

Figura 1 - Exemplo de questão objetiva utilizando escala.

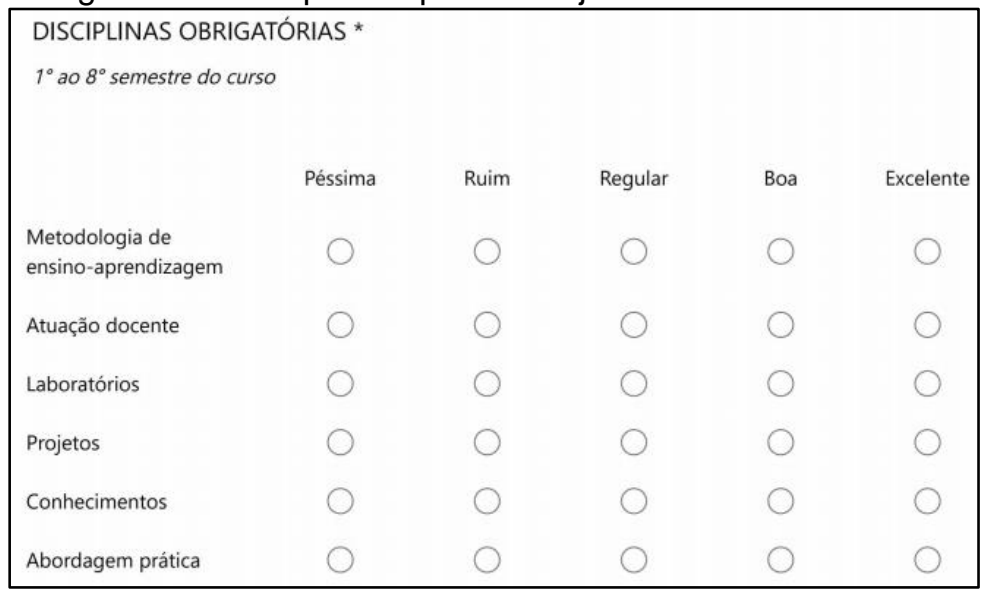

Fonte: Os autores (2021).

Figura 2 - Exemplo de questão objetiva utilizando escala.

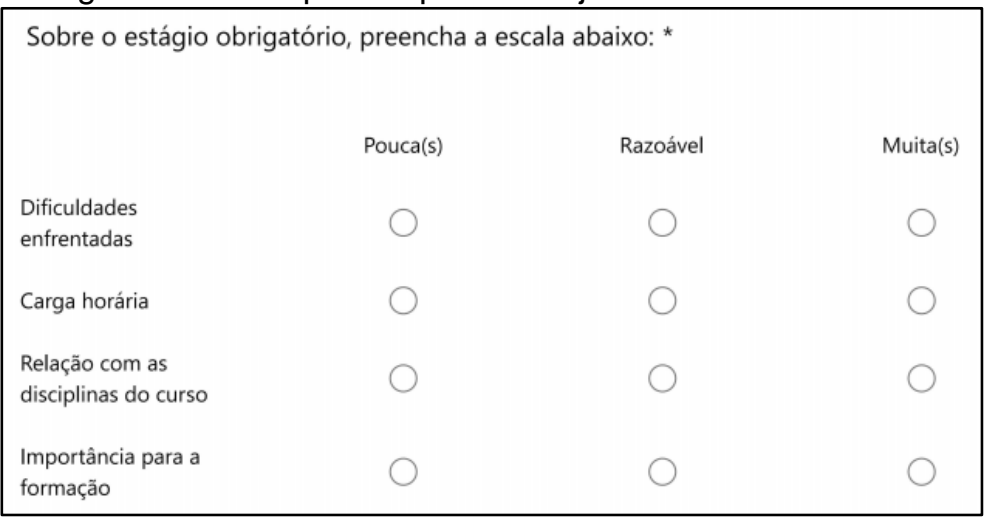

Fonte: Os autores (2021).

A realização ou não de algum tipo de especialização após a graduação foi questionada através da estrutura de caixa de seleção onde o respondente poderia marcar as opções de seu interesse (Figura 3). Isso também foi realizado para o oferecimento de projetos de ensino, pesquisa e extensão durante o período de formação. Outro aspecto avaliado foi a promoção de visitas técnicas enquanto eram acadêmicos dando-os a opção de classificá-las conforme a sua frequência (Figura 4).

O formulário procurou compreender o impacto das atividades extracurriculares realizadas na formação do acadêmico, bem como da sua participação em iniciativas da própria universidade onde também se fez uso de caixas de seleção. Outras questões abordadas no formulário foram relacionadas aos estágios (curricular supervisionado e nãocurricular) nos quais foram analisadas as eventuais dificuldades encontradas, como se deu a aquisição de conhecimento, 0 desenvolvimento de habilidades práticas e 0 aproveitamento dessa experiência pelo formado. 
Figura 3 - Exemplo de questão objetiva utilizando caixa de seleção.

Possui alguma especialização relacionada à área de formação? *
$\square$ Lato-sensu (MBA, especialização)
$\square$ Mestrado
$\square$ Doutorado
$\square$ Pós-doutorado
$\square$ Não possuo nenhuma especialização

Fonte: Os autores (2021).

Figura 4 - Exemplo de questão objetiva.

Foram oferecidas visitas técnicas durante o período de graduação? *
Sim, muitas.
Sim, mas eram raras.
Não.

Fonte: Os autores (2021).

Para a avaliação do currículo, criou-se uma estrutura de avaliação para cada grupo de disciplinas presentes no projeto pedagógico do curso a fim de avaliar os seguintes itens: metodologia de ensino-aprendizagem, atuação docente, laboratórios, projetos, conhecimentos e abordagem prática conforme ilustrado anteriormente pela Figura 1. O incentivo à utilização de softwares durante as atividades de formação do egresso também foi investigado no formulário. Além disso, o Trabalho de Conclusão de Curso (TCC) foi especificamente abordado a respeito do seu propósito, a temática utilizada, a orientação recebida pelo aluno e a atuação da banca avaliadora do trabalho.

As questões discursivas possibilitaram que o egresso compartilhasse suas opiniões a respeito da formação oferecida e suas experiências durante a trajetória acadêmica. Nesse sentido, foi questionado se o egresso atuava na área de formação do curso e a seguir qual era esta área, independente da primeira resposta. Também foi apresentada uma estrutura correspondente às disciplinas curriculares do $1^{\circ}$ ao $8^{\circ}$ semestre, a partir da qual questionouse sobre eventuais lacunas observadas na estrutura curricular e sugestões de reformulações (Figura 5).

Com o intuito de obter maior objetividade nas respostas, propôs-se a avaliação da formação proporcionada pelo curso de acordo com as principais áreas estabelecidas pelo CNPq para a Engenharia Civil (Figura 6). Assim, os egressos poderiam expressar suas opiniões relacionando as referidas áreas com a estrutura da Figura 5, que abrangeu as disciplinas através das siglas pelas quais eram conhecidas. 
Figura 5 - Exemplo de questão discursiva.

\begin{tabular}{|c|c|c|c|c|c|c|c|c|}
\hline & $\begin{array}{c}1^{\circ} \\
\text { SEMESTRE }\end{array}$ & $\begin{array}{c}2^{\circ} \\
\text { SEMESTRE }\end{array}$ & $\begin{array}{c}3^{\circ} \\
\text { SEMESTRE }\end{array}$ & $\begin{array}{l}4^{\circ} \\
\text { SEMESTRE }\end{array}$ & $\begin{array}{c}5^{\circ} \\
\text { SEMESTRE }\end{array}$ & $\begin{array}{l}6^{\circ} \\
\text { SEMESTRE }\end{array}$ & $\begin{array}{l}70^{\circ} \\
\text { SEMESTRE }\end{array}$ & $\begin{array}{l}8^{\circ} \\
\text { SEMESTRE }\end{array}$ \\
\hline $\begin{array}{l}\text { ForMACio } \\
\text { GRRAL }\end{array}$ & $\begin{array}{l}\text { IEC } \\
\text { SOR } \\
\text { SMA }\end{array}$ & $\begin{array}{l}\text { AlP } \\
\text { MEP }\end{array}$ & & & & & & DAE \\
\hline Fisica & & $\begin{array}{l}\text { FGEI } \\
\text { FEXI }\end{array}$ & $\begin{array}{l}\text { FGE II } \\
\text { FEXXII }\end{array}$ & FGE II & & & & \\
\hline MATEḾ̇̈CA & $\begin{array}{l}\text { CDII } \\
\text { GAN }\end{array}$ & $\frac{\mathrm{CDIII}}{\mathrm{ALI}}$ & $\begin{array}{l}\text { CVE } \\
\text { EDI }\end{array}$ & CAN & EST & & & \\
\hline $\begin{array}{l}\text { CONSTRUCío } \\
\text { CIVIL. }\end{array}$ & $\begin{array}{l}\text { DBA } \\
\text { QEEC }\end{array}$ & DAT & & $\begin{array}{l}\text { PAR } \\
\text { MCCI }\end{array}$ & $\begin{array}{c}\mathrm{MCCCI} \\
\mathrm{DPC}\end{array}$ & $\frac{C C C}{\mathrm{TECI}}$ & $\begin{array}{l}\text { EEC } \\
\text { TECII } \\
\text { SPRI }\end{array}$ & $\begin{array}{l}\text { PCO } \\
\text { SPR II } \\
\text { GCC }\end{array}$ \\
\hline $\begin{array}{c}\text { INFRA.E } \\
\text { TRANSPORTE }\end{array}$ & & & TOP I & TOP II & & ETTI & ETT II & \\
\hline $\begin{array}{l}\text { ESTRUTURAS } \\
\text { ENGESHARA } \\
\text { MDRAULICA }\end{array}$ & & & MGE & & $\begin{array}{l}\text { RMAII } \\
\text { TESI } \\
\text { HIDI }\end{array}$ & $\begin{array}{l}\text { CARI } \\
\text { TES II } \\
\text { HID II }\end{array}$ & $\begin{array}{c}\text { CAR II } \\
\text { HIA } \\
\text { SAA }\end{array}$ & EMM \\
\hline
\end{tabular}

Fonte: Os autores (2021).

Figura 6 - Exemplo de questão discursiva.

Segundo as áreas do CNPq abaixo, o que você teria a comentar a respeito da formação proporcionada pelo curso?

\section{Construção Civil \\ II. Engenharia Hidráulica \\ III. Estruturas \\ IV. Geotecnia \\ V. Infraestrutura e Transporte}

Fonte: Os autores (2021)

Também se investigou a formação empreendedora oferecida ou não pelo curso e quais seriam os tópicos considerados essenciais pelos egressos que não foram oferecidos no decorrer do curso. Ao final, foi solicitado que os alunos elencassem os pontos que consideraram fracos ou fortes do curso e avaliassem a sua própria atuação como discente durante a graduação (Figura 7).

Figura 7 - Questão auto avaliativa.

Na sua opinião, como foi a sua atuação como acadêmico durante a graduação na
UDESC? *

Fonte: Os autores (2021).

\section{ANÁLISE DAS PRINCIPAIS RESPOSTAS REGISTRADAS E CONCEPÇÃO DE AÇÕES PARA O CURSO}

As respostas obtidas foram registradas com o auxílio do próprio Microsoft Forms (Figura 8) e interpretadas individualmente pelos membros do NDE que desenvolveram o formulário. 
Figura 8 - Exemplo de cadastro dos egressos.

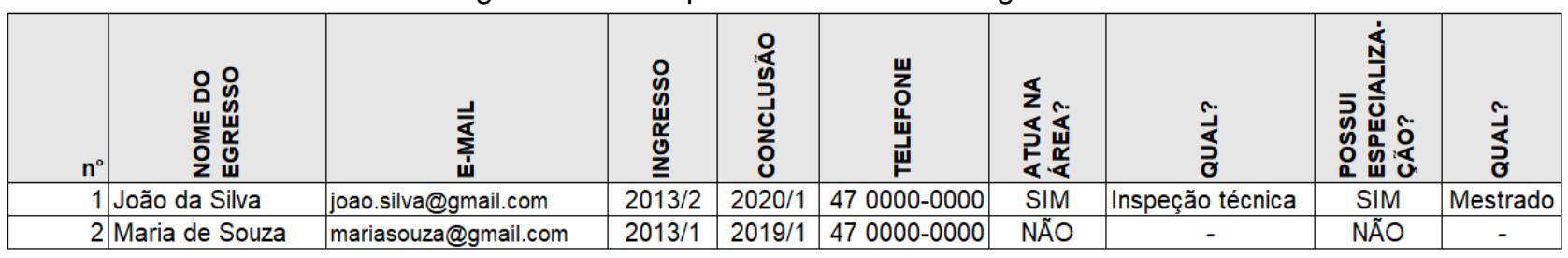

Fonte: Os autores (2021).

Aproximadamente $71 \%$ dos respondentes afirmaram que a sua área de atuação profissional estava inserida na área de formação do curso. Já a respeito das especializações relacionadas à área de formação, quase $61 \%$ responderam que não possuem nenhuma especialização, enquanto aproximadamente $25 \%$ afirmaram ter realizado ao menos uma especialização lato sensu e 15\% assinalaram que obtiveram 0 grau de mestre após a conclusão da graduação. Isso demonstra que ainda é a minoria que compreende a importância do constante aperfeiçoamento após a graduação.

$\mathrm{Em}$ seguida, quando solicitado sobre as atividades oferecidas durante o seu processo de formação, dentre elas ensino, pesquisa e extensão, as respostas revelaram que apenas 35\% afirmaram terem sido oferecidos simultaneamente projetos de ensino, pesquisa e extensão. Ainda, $18 \%$ assinalaram terem sido oferecidos apenas projetos de extensão; 9\% para apenas projetos de pesquisa e, $7 \%$ para apenas projetos de ensino durante o seu período de graduação. Isto ressalta a necessidade de fomentar as iniciativas de ensino, pesquisa e extensão num curso de graduação através do envolvimento de um número maior de docentes neste âmbito, além da necessidade da melhor divulgação das atividades já existentes.

Sobre a promoção de visitas técnicas durante o período de graduação, $88 \%$ dos alunos responderam que as visitas, apesar de oferecidas, eram raras. Tendo em vista 0 objetivo de aproximar o acadêmico do ambiente profissional e da necessidade de uma abordagem prática faz-se imprescindível tratar as visitas técnicas como um componente do planejamento das disciplinas e do próprio departamento.

A realização de estágio extracurricular, também conhecido como estágio nãoobrigatório teve resultados interessantes. Quando questionados se tiveram a oportunidade de realizar tal modalidade de estágio, mais de $80 \%$ dos respondentes afirmaram que sim. Contudo, nas questões discursivas foi massivamente relatada a dificuldade em se realizar esta atividade. A principal dificuldade apontada nas respostas foi de conciliar esta atividade com a disponibilização de horários de aulas proposta durante a graduação. A seguir, quando solicitado que classificassem essa experiência (se realizada), as respostas referentes aquisição de conhecimento, desenvolvimento de experiência profissional e habilidades práticas foi excelente para mais de $60 \%$ dos respondentes. Este resultado ressalta a importância do estágio extracurricular e demanda incentivo por parte da universidade a esta prática através do contato contínuo com o mercado de trabalho e o setor produtivo.

A formação básica proporcionada pelo curso, que se entende por aquela abrangida pelas disciplinas de matemática, física, química e ciências humanas, foi avaliada pelos egressos conforme Tabela 1. Nas respostas abertas foi mencionado o uso de problemas abstratos dentro das disciplinas de formação básica em detrimento da utilização de problemas reais e contextualizados para as áreas da Engenharia Civil. Além disso, as respostas abertas sugeriram implementar abordagens de ensino que desenvolvessem as soft skills como o relacionamento interpessoal, gestão de pessoas e comunicação. Neste 
sentido, ressalta-se que as habilidades transversais podem ir se aprofundando ao longo do curso, independente das áreas de conhecimento.

Tabela 1 - Resultados para formação básica

\begin{tabular}{c|c|c|c|c|c}
\hline & Péssima & Ruim & Indiferente & Boa & Excelente \\
\hline Matemática & - & $2,3 \%$ & $11,3 \%$ & $\mathbf{4 8 , 1} \%$ & $38,3 \%$ \\
\hline Química & $4,5 \%$ & $13,5 \%$ & $\mathbf{4 4 , 4} \%$ & $31,6 \%$ & $6,0 \%$ \\
\hline Física & - & $3,0 \%$ & $13,5 \%$ & $\mathbf{5 7 , 9 \%}$ & $25,6 \%$ \\
\hline Ciências humanas & $4,5 \%$ & $20,3 \%$ & $\mathbf{4 3 , 6} \%$ & $24,1 \%$ & $7,5 \%$ \\
\hline
\end{tabular}

Fonte: Os autores (2021).

A Tabela 2 apresenta os resultados para a formação das disciplinas obrigatórias que compõem $75 \%$ da carga horária do curso. Nota-se que os conhecimentos transmitidos e a atuação docente possuem resultados melhores que a metodologia ensino-aprendizagem, projetos, laboratórios e, principalmente, da abordagem prática. Em respostas abertas, a abordagem prática foi elencada como o principal ponto fraco do curso. Isso evidencia a necessidade de investimentos em laboratórios, o redesenho das disciplinas e planos de ensino de maneira a proporcionar maior integração entre teoria e prática envolvendo atividades voltadas à aplicação de conhecimentos.

Tabela 2 - Resultados para formação das disciplinas obrigatórias.

\begin{tabular}{c|c|c|c|c|c}
\hline & Péssima & Ruim & Indiferente & Boa & Excelente \\
\hline $\begin{array}{c}\text { Metodologia de } \\
\text { ensino-aprendizagem }\end{array}$ & $1,5 \%$ & $7,5 \%$ & $\mathbf{4 9 , 6} \%$ & $36,1 \%$ & $5,3 \%$ \\
\hline Atuação docente & - & $6,8 \%$ & $34,6 \%$ & $\mathbf{5 1 , 9 \%}$ & $6,8 \%$ \\
\hline Laboratórios & $3,0 \%$ & $21,1 \%$ & $\mathbf{4 3 , 6} \%$ & $28,6 \%$ & $3,8 \%$ \\
\hline Projetos & $3,0 \%$ & $19,5 \%$ & $\mathbf{4 4 , 4 \%}$ & $27,1 \%$ & $7,5 \%$ \\
\hline Conhecimentos & $0,8 \%$ & - & $20,3 \%$ & $\mathbf{5 9 , 4 \%}$ & $19,5 \%$ \\
\hline Abordagem prática & $15,8 \%$ & $32,3 \%$ & $\mathbf{3 8 , 3} \%$ & $12,8 \%$ & $0,8 \%$ \\
\hline
\end{tabular}

Fonte: Os autores (2021).

O PPC vigente do curso de Engenharia Civil do CCT/UDESC conta com duas ênfases a serem escolhidas (Engenharia Urbana e Sustentabilidade na Construção Civil), sendo que pelo pequeno interesse notado por parte da comunidade acadêmica, esta última deixou de ser oferecida. Também conta com um elenco de disciplina optativas de formação geral (Grupo I) e outro com disciplinas relacionadas especificamente a projetos (Grupo II), que também foram abordadas no questionário.

A avaliação das disciplinas optativas do curso (Tabelas 3 e 4) acompanhou a tendência das respostas anteriores, mas obtendo resultados inferiores nos itens pertinentes ao uso de laboratórios e à realização de projetos. As respostas referentes ao Grupo II de disciplinas optativas apresentaram resultados um tanto diferentes neste item devido ao escopo de projetos presente neste arranjo de disciplinas (Tabela 5). A indiferença no item metodologia de ensino-aprendizagem reflete a importância de questioná-las e planejar uma reformulação na maneira como o ensino é realizado dentro da universidade. Muitas são as vezes que os próprios alunos desconhecem os diferentes tipos de metodologia de ensinoaprendizagem, já que a metodologia de aula expositiva clássica é a predominante no curso. É preciso repensar a vasta utilização de aulas expositivas ao longo do curso, que por vezes não oportunizavam novas experiências de aprendizagem indispensáveis à formação do estudante. 
Tabela 3 - Resultados para formação das disciplinas optativas de ênfase em Engenharia Urbana.

\begin{tabular}{c|c|c|c|c|c}
\hline & Péssima & Ruim & Indiferente & Boa & Excelente \\
\hline $\begin{array}{c}\text { Metodologia de } \\
\text { ensino-aprendizagem }\end{array}$ & $3,9 \%$ & $12,4 \%$ & $\mathbf{3 8 , 0} \%$ & $\mathbf{3 8 , 0} \%$ & $7,8 \%$ \\
\hline Atuação docente & $3,1 \%$ & $7,8 \%$ & $38,8 \%$ & $\mathbf{4 0 , 3} \%$ & $10,1 \%$ \\
\hline Laboratórios & $16,3 \%$ & $31,8 \%$ & $\mathbf{3 9 , 5} \%$ & $11,6 \%$ & $0,8 \%$ \\
\hline Projetos & $5,5 \%$ & $25,8 \%$ & $\mathbf{3 7 , 5} \%$ & $25,8 \%$ & $5,5 \%$ \\
\hline Conhecimentos & $3,9 \%$ & $6,3 \%$ & $35,4 \%$ & $\mathbf{4 1 , 7 \%}$ & $12,6 \%$ \\
\hline Abordagem prática & $9,3 \%$ & $24,8 \%$ & $\mathbf{3 9 , 5} \%$ & $23,3 \%$ & $3,1 \%$ \\
\hline
\end{tabular}

Fonte: Os autores (2021).

Tabela 4 - Resultados para formação das disciplinas optativas do Grupo I.

\begin{tabular}{c|c|c|c|c|c}
\hline & Péssima & Ruim & Indiferente & Boa & Excelente \\
\hline $\begin{array}{c}\text { Metodologia de } \\
\text { ensino-aprendizagem }\end{array}$ & $4,5 \%$ & $15,0 \%$ & $\mathbf{4 2 , 9} \%$ & $34,6 \%$ & $3,0 \%$ \\
\hline Atuação docente & $6,0 \%$ & $12,0 \%$ & $\mathbf{3 8 , 3} \%$ & $35,3 \%$ & $8,3 \%$ \\
\hline Laboratórios & $19,5 \%$ & $29,3 \%$ & $\mathbf{4 2 , 1} \%$ & $8,3 \%$ & $0,8 \%$ \\
\hline Projetos & $7,5 \%$ & $23,3 \%$ & $\mathbf{4 2 , 1} \%$ & $24,1 \%$ & $3,0 \%$ \\
\hline Conhecimentos & $4,5 \%$ & $9,8 \%$ & $33,8 \%$ & $\mathbf{4 2 , 1} \%$ & $9,8 \%$ \\
\hline Abordagem prática & $12,8 \%$ & $21,1 \%$ & $\mathbf{4 0 , 6} \%$ & $22,6 \%$ & $3,0 \%$ \\
\hline
\end{tabular}

Fonte: Os autores (2021).

Tabela 5 - Resultados para formação das disciplinas optativas do Grupo II.

\begin{tabular}{c|c|c|c|c|c}
\hline & Péssima & Ruim & Indiferente & Boa & Excelente \\
\hline $\begin{array}{c}\text { Metodologia de } \\
\text { ensino-aprendizagem }\end{array}$ & $3,0 \%$ & $10,5 \%$ & $\mathbf{4 3 , 6} \%$ & $37,6 \%$ & $5,3 \%$ \\
\hline Atuação docente & $0,8 \%$ & $9,8 \%$ & $38,3 \%$ & $\mathbf{3 9 , 8} \%$ & $11,3 \%$ \\
\hline Laboratórios & $19,5 \%$ & $24,8 \%$ & $\mathbf{4 8 , 1} \%$ & $6,0 \%$ & $1,5 \%$ \\
\hline Projetos & $5,3 \%$ & $18,8 \%$ & $\mathbf{3 7 , 6} \%$ & $30,1 \%$ & $8,3 \%$ \\
\hline Conhecimentos & $1,5 \%$ & $8,3 \%$ & $25,6 \%$ & $\mathbf{4 7 , 4 \%}$ & $17,3 \%$ \\
\hline Abordagem prática & $5,3 \%$ & $23,3 \%$ & $\mathbf{3 6 , 1} \%$ & $27,1 \%$ & $8,3 \%$ \\
\hline
\end{tabular}

Fonte: Os autores (2021).

Uma parcela significativa dos egressos apontou que existe dificuldade em unir os conhecimentos básicos aos específicos, ocorrendo assim ausência de integração entre os campos de conhecimento. Este apontamento é um problema reconhecido do ensino em engenharia pois se parte do pressuposto que o aluno, uma vez em contato com diversos domínios de conhecimento em diferentes escalas de tempo, seja plenamente capaz de integrá-los sem muita dificuldade.

Como é amplamente percebido, esta integração não ocorre e é imprescindível enxergar o profissional formado como alguém apto, "competente" àquilo que lhe é atribuído. Dessa forma, o resultado do processo de formação deve ser uma construção de conhecimentos, composição de pequenas partes de competências que ao final configuram o que se espera de um engenheiro do século XXI. Por fim, quando questionados se a metodologia de ensino utilizada no curso os preparou para os desafios enfrentados na prática profissional, aproximadamente $50 \%$ afirmaram que sim, $20 \%$ se mantiveram indiferentes e 30\% afirmaram que não foram preparados para os desafios da sua profissão.

A elaboração de um projeto integrado orientado nas fases finais do curso poderia contribuir na articulação dos conhecimentos construídos ao longo da formação do estudante. Nesta modalidade poderiam ser aplicadas diversas áreas de conhecimento (arquitetura, estruturas, fundações, instalações prediais, entre outras) abrangendo inclusive, a compatibilização de projetos, que foi imensamente citada nas respostas 
abertas. Além disso, seria uma oportunidade de utilização de softwares com o devido acompanhamento dos docentes orientadores.

Sobre as ações do departamento responsável pelo curso no incentivo a uma formação empreendedora, os respondentes afirmaram ser algo extremamente necessário, porém foi praticamente inexistente. Diante disso, sugeriram aprimorar o contato com entidades empresariais, reforçando o fomento às empresas juniores, realização de palestras de profissionais das diversas áreas de atuação e oportunidade de ex-alunos contribuírem com o curso através do compartilhamento de suas experiências no mercado de trabalho, carreira acadêmica e demais áreas.

De modo geral, os egressos apontaram as poucas atividades práticas e baixo incentivo ao uso de softwares e à abordagem computacional dentro do escopo dos problemas de engenharia. Contudo, ressaltaram terem obtido uma ótima formação matemática e o desenvolvimento de um forte raciocínio lógico durante o curso. Ainda, acrescentaram que o curso forma profissionais determinados e resilientes frente às adversidades da profissão.

\section{CONSIDERAÇÕES FINAIS}

O presente estudo apresentou os resultados provenientes de um formulário de autoavaliação do curso, aplicado aos egressos formados no período de 2016/1 até 2020/1. Mais de $40 \%$ dos 302 egressos responderam ao formulário fazendo com que a amostragem obtida tivesse relevância estatística com relação à população.

Os resultados indicaram as principais falhas e os principais acertos do curso, através do ponto de vista do egresso. Dentre as maiores falhas estão a falta de laboratórios adequados e poucas visitas técnicas, assim como a falta de incentivo ao uso de softwares e o pouco desenvolvimento do empreendedorismo dentro do curso.

Já como pontos fortes estão a aquisição de conhecimentos ao longo da formação e uma boa atuação dos professores em suas atividades acadêmicas, sejam elas na abordagem das disciplinas ou na orientação de TCCs. Também, identificou-se que grande parte dos respondentes (70\%) está satisfeita com a formação obtida no curso corroborando com a grande maioria (71\%) que atua profissionalmente na sua área de formação.

As informações obtidas são muito valiosas para o aperfeiçoamento do curso, pois evidenciam problemas pontuais. De qualquer forma, esta é apenas uma ferramenta para que sejam levadas ao NDE informações relevantes na estruturação do PPC e não deve ser considerada como verdade absoluta, tendo em vista somente a participação dos egressos recentemente formados pelo curso.

\section{REFERÊNCIAS}

CONSELHO NACIONAL DE EDUCAÇÃO. Resolução CNE/CES n 2: Institui as Diretrizes Curriculares Nacionais do Curso de Graduação em Engenharia. Brasília, 2019.

\section{DEPARTAMENTO DE ENGENHARIA CIVIL UDESC/CCT. Projeto Pedagógico do} Curso. Joinville, 2008.

MARINHO-ARAUJO, Claisy. Maria; RABELO, Mauro Luiz. Avaliação educacional: a abordagem por competências. Avaliação: Revista da Avaliação da Educação Superior (Campinas), v. 20, n. 2, p. 443-466, 2015. 
PRADO, Fábio do; SANTOS, Roberto Baginski B. Novas DCNs dos cursos de graduação em Engenharia e a perspectiva da avaliação centrada em competências. In: OLIVEIRA, V. F. (org.). A engenharia e as novas DCNs: oportunidades para formar mais e melhores engenheiros. Rio de Janeiro: LTC, 2019. p. 219-225.

\title{
PROFILE OF CIVIL ENGINEERING GRADUATES FROM UDESC/CCT: THE IMPORTANCE IN THE REFORMULATION OF THE PPC
}

\begin{abstract}
For an undergraduate course to reach its competencies development objectives, an integrated curriculum must be conceived through collectivity. This way, it is essential to gather the course data in order to obtain the technical and didactic formation demands to support this new curriculum to be implemented. The present paper aimed to investigate, under the graduate's perspective, the strengths and weaknesses in the CCT/UDESC's Civil Engineering Course. To do so, the Structuring Teaching Group (STG), prepared a survey that was applied to the graduates between 2016/1 and 2020/1. This survey was answered by $44 \%$ of the graduates. Being registered the responses, a database was developed with information on the graduates and produced a feedback panel to help the SDC to conceive the new pedagogic course project. Throughout the activities described in this paper, it was perceived the need of information on the graduates of engineering courses, and then, was confirmed the relevancy of the present research.
\end{abstract}

Keywords: Education in Engineering. National Curriculum Guidelines. Pedagogical Project. Profile of Graduates. 